Questions vives

\section{Questions Vives}

Recherches en éducation

Vol. $6 n^{\circ} 12$ | 2009

Evaluer les enseignants et les formateurs. Comment ? Pourquoi ? Pour quoi ?

\title{
Construction d'un dispositif d'évaluation des enseignements et des formations : le point de vue des apprenants
}

Christine Gangloff, Marc Weisser, Saloua Bennaghmouch et SondessBen Abid-Zarrouk

\section{CpenEdition}

\section{Journals}

Édition électronique

URL : http://journals.openedition.org/questionsvives/407

DOI : $10.4000 /$ questionsvives.407

ISBN : 978-2-8218-1084-6

ISSN : 1775-433X

Éditeur

Université Aix-Marseille (AMU)

Édition imprimée

Date de publication : 6 juin 2009

Pagination : 61-76

ISBN : 978-2-912643-36-0

ISSN : $1635-4079$

Référence électronique

Christine Gangloff, Marc Weisser, Saloua Bennaghmouch et Sondess-Ben Abid-Zarrouk, "Construction d'un dispositif d'évaluation des enseignements et des formations : le point de vue des apprenants », Questions Vives [En ligne], Vol.6 n¹2 | 2009, mis en ligne le 01 janvier 2011, consulté le 19 avril 2019. URL : http://journals.openedition.org/questionsvives/407 ; DOI : 10.4000/ questionsvives. 407

Questions Vives est mis à disposition selon les termes de la licence Creative Commons Attribution Pas d'Utilisation Commerciale - Pas de Modification 4.0 International. 


\title{
Construction d'un dispositif d'évaluation des enseignements et des formations : le point de vue des apprenants
}

\author{
Christine Gangloff 1 , Marc Weisser ${ }^{2}$, \\ Saloua Bennaghmouch ${ }^{3}$, Sondess Ben Abid-Zarrouk ${ }^{4}$
}

Résumé: L'université française a l'habitude d'évaluer ses étudiants. Mais l'exigence d'évaluer les systèmes de formation eux-mêmes apparaît progressivement. Le but est d'une part d'améliorer la qualité des enseignements, et d'autre part de permettre la comparaison des systèmes éducatifs. Dans ce contexte, notre recherche consiste à recueillir le point de vue des étudiants sur les critères de qualité d'une formation. Nous avons procédé à des entretiens sous forme de focus groups. Nous avons interrogé un panel représentatif de l'ensemble des dispositifs: formation générale / formation professionnelle; UFR / IUT / Écoles d'Ingénieurs; en début de formation / en fin de formation. Nous avons de la sorte pu identifier les positions constantes d'un groupe à l'autre, ainsi que les avis complémentaires.

Mots-clés : évaluation des formations, critères de qualité, université, focus group.

Abstract: One of the main missions of French university is to evaluate students. But the requirement to assess also training systems is gradually growing. The goal is, on the one hand, to improve the quality of courses and training, and, on the other hand, to allow the comparison of educational systems. In this context, our research consists in collecting the students' point of view about the quality standards they look for during their training. We organized interviews using the methodology of focus groups. We questioned a representative panel of students: general training / vocational training; UFR / IUT / Schools of Engineers; at the beginning of their training / at the end of their training. Then, we identified the constant positions from one group to another, as well as complementary opinions.

Keywords: training evaluation, quality standards, university, focus group.

\footnotetext{
${ }^{1}$ Maître de conférences en sciences de l'éducation. Université de Haute-Alsace, Laboratoire Interuniversitaire des Sciences de l'Éducation et de la Communication (LISEC - EA 2310).

2 Professeur en sciences de l'éducation. Université de Haute-Alsace, LISEC.

${ }^{3}$ Maître de conférences en sciences économiques. Université de Haute-Alsace, LISEC.

${ }^{4}$ Maître de conférences en sciences de l'éducation. Université de Haute-Alsace, LISEC.
} 


\section{Construction d'un dispositif d'évaluation des enseignements et des formations : le point de vue des apprenants.}

\section{Problématique}

La mise en place d'une évaluation des enseignements et des formations est une obligation maintes fois affirmée par les textes relatifs aux universités même si les finalités ont pu évoluer dans le temps. L'Université française exerce, sans aucun doute, son rôle d'évaluateur vis-à-vis des étudiants. Mais elle est moins habituée à se trouver dans la situation d'être évaluée et des résistances subsistent.

D'abord tournée vers la mesure des résultats des politiques publiques, l'évaluation a été ensuite orientée en 1992 vers les enseignements en se fondant sur l'appréciation des étudiants. En 1997, elle s'est étendue également aux formations mais en se fondant toujours exclusivement sur l'appréciation des étudiants avec pour objectif de connaître leur avis sur les éléments pédagogiques, pour l'enseignant, et sur l'organisation des études, pour l'institution. En 2002, le but affirmé de l'évaluation dépasse le simple constat d'une situation pour être envisagé comme un facteur de progrès dans le dessein d'améliorer les dispositifs pédagogiques et l'apprentissage grâce au dialogue entre les équipes de formation et les étudiants. D'une évaluation sommative, l'Université passe à une évaluation formative. L'appréciation des apprenants n'est plus l'élément unique de référence mais ils doivent néanmoins être associés à la mise en place du dispositif.

Parallèlement, dans le cadre de l'Espace Européen de l'Enseignement Supérieur (EEES), une nouvelle dimension apparaît dont l'enjeu est la comparabilité des formations afin de favoriser la mobilité des étudiants. II s'agit alors de mettre en place des systèmes de garantie de la qualité externe tout en poursuivant le développement des systèmes de garantie interne.

Sur le plan méthodologique, cela fait apparaître deux types d'évaluations : celles menées sur la base d'un référentiel interne qui contribue à l'amélioration de la qualité des formations et des enseignements et à la responsabilisation des universités; celles conduites à partir d'un référentiel externe du type certification qui permet, par un système d'assurance qualité, la comparaison des formations entre elles et vise à rassurer, d'une part, les usagers (utilisateurs des enseignements et des formations) et, d'autre part, les employeurs ou, dans l'hypothèse d'une mobilité étudiante, d'autres universités (utilisateurs des formations).

\section{Contexte}

II convient préalablement de resituer cette démarche dans le cadre plus global de la mise en place d'un système de management interne de la qualité au sein de l'Université de HauteAlsace (France). Dans le domaine de l'évaluation des enseignements et des formations, le choix a été fait de se baser sur le réérentiel 1 de l'ENQA ${ }^{5}$ correspondant à une analyse critériée interne qui pourra à terme évoluer vers une analyse externe.

Ce référentiel liste sept références qui sont réputées traduire les attentes de la société vis-à-vis des établissements d'enseignement supérieur, proposant ainsi une approche globale de la qualité des enseignements et des formations :

\footnotetext{
${ }^{5}$ European Association for Quality Assurance in Higher Education.
} 
Evaluer les enseignants et les formateurs

Comment, pourquoi, pour quoi ?

\begin{abstract}
- Politique et procédures pour le management de la qualité : les établissements doivent avoir une politique et des procédures associées pour le management de la qualité et des niveaux de leurs programmes et de leurs diplômes. Ils doivent également s'engager explicitement dans l'instauration d'une culture qui reconnaisse l'importance de la qualité et de son management. Pour y parvenir, les établissements doivent mettre en œuvre et développer une stratégie visant à l'amélioration continue de la qualité. La stratégie, la politique et les procédures doivent avoir un statut officiel et être rendues publiques. Elles doivent prévoir un rôle pour les étudiants et les autres parties intéressées.

- Approbation, examen et révision périodique des programmes et des diplômes: les établissements doivent disposer de mécanismes officiels pour l'approbation, l'examen et la révision périodique de leurs programmes et de leurs diplômes.

-Évaluation des étudiants : les étudiants doivent être évalués en fonction de critères, règlements et procédures publics, appliqués de manière systématique et constante.

- Management de la qualité du corps enseignant : les établissements doivent avoir les moyens de s'assurer de la qualité et de la compétence de leur corps enseignant. Ces moyens doivent être à la disposition des équipes d'évaluation externe et faire l'objet de commentaires dans les rapports d'évaluation.

- Outils pédagogiques et soutien des étudiants : les établissements doivent s'assurer que les ressources affectées aux outils pédagogiques et au soutien des étudiants sont adéquates et adaptées à chaque programme proposé.

- Systèmes d'information : les établissements doivent garantir qu'ils collectent, analysent et se servent des informations nécessaires au pilotage efficace de leurs programmes de formation et autres activités.

- Information du public: les établissements doivent régulièrement rendre publiques des informations à jour, impartiales et objectives, à la fois quantitatives et qualitatives, sur les programmes et les diplômes qu'ils proposent.
\end{abstract}

Afin de bien délimiter la portée de ces éléments, nous rappellerons utilement la définition de la notion de standard retenue par l'ENQA :

"Les standards sont définis comme des références générales décrivant des politiques et des pratiques destinées à s'assurer que des niveaux prédéfinis et explicites de qualité sont atteints dans la conception, la délivrance et l'évaluation des cours et des diplômes d'enseignement supérieur. Un standard ne dit pas comment un résultat doit être atteint, il dit simplement ce que devrait être ce résultat. Le domaine d'application et la manière d'utiliser les standards sont déterminés en fonction des contextes particuliers des établissements et des systèmes nationaux ; à mesure que ceux-ci évoluent, il peut être nécessaire de modifier les domaines d'application et les manières d'utiliser les standards $» .6$

Comme le souligne Bruno Curvale, président de l'ENQA, les démarches qualité des établissements doivent satisfaire leurs besoins propres, mais aussi les attentes de leurs partenaires et usagers. Le référentiel 1 précise les objectifs à atteindre. Des lignes directrices

\footnotetext{
${ }^{6}$ [http://www.cne-evaluation.fr/fr/present/interstandardsENQA.htm].
} 
précisent l'esprit des références et, le cas échéant, suggèrent des pratiques possibles. ${ }^{7}$ Elles sont définies " comme des explications non prescriptives de l'importance des standards ". 8

Par conséquent, quand les objectifs sont connus, des outils peuvent être construits en interne pour répondre aux spécificités et stratégies propres à chaque établissement.

L'objet de cet article est de présenter l'un des aspects de cette démarche consistant à analyser la ligne directrice prévoyant la nécessité pour les enseignants de bénéficier d'un retour d'appréciation sur la qualité de leurs activités d'enseignement. Cette ligne directrice peut être associée à la quatrième référence relative au management de la qualité du corps enseignant qui mentionne que "les établissements doivent avoir les moyens de s'assurer de la qualité et de la compétence de leur corps enseignant». Cet article a pour finalité de contribuer à la réflexion sur l'implication des étudiants dans l'évaluation des enseignements et des formations la mettant ainsi en relation avec leurs retours d'appréciation sur les activités d'enseignement.

En effet, la première référence nous rappelle également la nécessaire participation des étudiants au management de la qualité des enseignements et des formations. Dans ce même sens, Murray (1984) souligne que "les étudiants sont les seuls témoins constants de la prestation d'enseignement, ils sont les seuls à pouvoir indiquer si les exposés du professeur sont clairs, si les commentaires sur les travaux les aident à progresser, si les documents utilisés favorisent la compréhension ».

Mais une démarche d'évaluation des enseignements et des formations doit d'évidence être abordée en tenant compte des réflexions antérieurement menées au sein des filières de formation. Ainsi, à partir du bilan exploratoire de la situation, effectué au sein des différentes UFR et du service commun de formation continue de l'Université de Haute-Alsace, nous avons relevé que cette évaluation, lorsqu'elle était mise en place, était faite sur la base de questionnaires de satisfaction, tous différents, élaborés dans la plupart des cas par les équipes pédagogiques elles-mêmes, sans y associer les apprenants.

Dans ces conditions, au niveau de l'établissement, l'UHA s'est située au niveau 1 du Plan Annuel de Performance (PAP) :

"Pour chacun de ces secteurs, l'établissement qualifie son degré d'avancement pour la mise en place d'une démarche d'auto-évaluation en choisissant une des cotations suivantes :

\footnotetext{
0 II n'y a pas d'action ou de projet significatif contribuant à ce but.

1 Un plan d'action a été défini et mis en œuvre.

2 La définition d'un plan d'action, sa mise en œuvre, le suivi et l'adaptation de sa réalisation 2 s'intègrent dans un cycle permanent (processus d'amélioration continue).

3 Le dispositif d'assurance qualité est validé par une instance externe reconnue.
}

II est convenu qu'une situation donnée correspond à une cotation lorsque cette caractéristique s'applique à plus de $80 \%$ du secteur concerné $" .9$

\footnotetext{
${ }^{7}$ Curvale B. (2006). ENQA dans l'Espace européen de l'enseignement supérieur, Bruxelles, 18 décembre 2006 [www.aeqes.be/documents/AEQES_18.12.06._Bruno\%20Curvale.ppt].

8 [http://www.cne-evaluation.fr/fr/present/interstandardsENQA.htm].
} 
Evaluer les enseignants et les formateurs

Comment, pourquoi, pour quoi ?

Nous pourrions cependant reprendre à notre compte la remarque de François Marie Gérard (2002) : «À aucun moment, on ne s'est demandé pourquoi on faisait cette évaluation (quel type de décision doit-on prendre au terme du processus ?), quels étaient les critères qui permettraient de fonder la décision, quels étaient les indicateurs nécessaires pour confronter la réalité à ces critères, quelle était la stratégie la plus appropriée pour recueillir l'information sur ces indicateurs, et enfin on n'a pas cherché à vraiment donner du sens aux résultats recueillis 》.

Quoi qu'il en soit, l'analyse de la situation actuelle laisse clairement apparaître une lacune dans la mise en place d'un système d'évaluation des enseignements et des formations. Et, s'il est utile que chaque composante d'une université mène une réflexion en la matière, il est nécessaire également d'envisager très rapidement une démarche globale visant à proposer des outils et un accompagnement, afin d'aider à la mise en place d'une auto-évaluation, en cohérence avec les objectifs stratégiques de l'établissement. Nous sommes invités, à travers ce processus, à faire évoluer nos modes de fonctionnement pour construire des dispositifs d'amélioration permanente et ainsi " accroître la responsabilité de nos établissements dans la mesure de la qualité de leur contribution au développement de la connaissance et de sa diffusion $" .10$

Cet état des lieux va dans le sens de l'affirmation de Detroz (2007) qui relève que, parmi les différents types d'évaluation envisageables, les questionnaires visant à recueillir l'avis des étudiants, apparaissent comme les plus usités au niveau mondial. Et par l'analyse des critères de qualité d'une évaluation et des objets qu'elle est susceptible d'appréhender, il met en exergue l'intérêt d'une telle méthode même si aucun système existant ne permet, isolément, d'appréhender toutes les facettes des actions didactiques.

II paraît utile à ce stade de rappeler le cadre théorique dans lequel s'inscrit la question de l'évaluation des enseignements.

\section{L'évaluation de la qualité des enseignements et des formations : choix théoriques}

Évaluer la qualité des enseignements et des formations suppose dans un premier temps de lever les ambiguités sur le sens à donner aux termes utilisés.

Premièrement, les enseignements visés correspondent à des cours ou des ensembles de cours agrégés par modules ou unités d'enseignement selon la finesse d'analyse recherchée. Ils présentent une double caractéristique. L'enseignement s'analyse d'abord comme un service, co-construit par l'enseignant et l'apprenant. En effet, " dans le domaine des services, le consommateur du service est co-producteur du service lui-même " (Aballéa \& Bercovitz, 1990) parce que celui-ci est le résultat d'une interaction avec le bénéficiaire qui doit participer à sa réalisation d'une manière plus ou moins soutenue. II a un rôle actif qui impacte fortement le résultat du processus. Cela conditionne la réussite du processus d'apprentissage et, vraisemblablement, le niveau de la satisfaction perçue. La deuxième propriété d'un service, qui peut aisément être déduite de la précédente, est qu'il ne peut par conséquent être

\footnotetext{
9 [http://edges.sup.adc.education.fr/edes/extranet/edes/extranet/contrats/editorial_contrats/autoeval/aut oE.htm]

10 Intervention de J.-M. Monteil, De Berlin à Bergen : nouveaux enjeux de l'évaluation, CNE, Dijon, Juin 2004.
} 
entièrement contrôlé a priori: les ingénieries pédagogiques (que nous appellerons ici dispositifs) doivent ensuite être mises en situation, avec les étudiants.

Par conséquent, l'évaluation de l'enseignement ne peut intervenir qu'a posteriori et doit porter sur deux domaines puisqu'à l'action d'enseigner doit répondre l'action d'apprendre. Cela concerne, d'une part, la pratique de l'enseignement ou teaching et d'autre part, la capacité des étudiants à apprendre et la façon dont l'enseignement est reçu ou learning (Rapport EVALUE 1998).

Nous appellerons de la même façon "formation" un ensemble cohérent d'enseignements ou d'UE, s'étendant sur un semestre (cas des mobilités d'étudiants), ou sur une, deux, voire trois années (cas des formations diplômantes).

L'évaluation se définit quant à elle comme "un acte délibéré et socialement organisé aboutissant à la production d'un jugement de valeur » (Barbier 1985). A la suite de Maurice Baslé (2000), nous relèverons qu'évaluer, c'est d'abord connaître, mais parce que le doute peut persister après l'évaluation, c'est plus encore " construire ensemble du sens " (Hoppe 1999).

Enfin, l'idée de qualité mérite d'être précisée. La norme ISO 9000:2000 la définit comme l'aptitude d'un ensemble de caractéristiques intrinsèques à satisfaire des exigences. Dans le domaine marchand, c'est la capacité d'un produit à satisfaire les clients en particulier par le respect des engagements pris (Caby \& Jambart, 2002). Cependant, ces modèles n'apparaissent pas toujours adaptés dans ce cadre parce qu'ils "sont des modèles de gestion de la qualité, mais pas d'évaluation de la qualité et sont fondés sur des liens de causalité qui ne sont pas démontrés ". ${ }^{11}$

De façon plus spécifique, l'ENQA avait proposé en 2004 la définition suivante de la qualité dans l'enseignement supérieur : "Description de l'efficacité de tout ce qui est entrepris pour s'assurer que les étudiants impliqués dans leurs études peuvent tirer un bénéfice maximum des possibilités de formation qui leur sont offertes et satisfont ainsi aux conditions d'obtention des diplômes qu'ils ambitionnent ".12 Mais cette notion de bénéfice maximum est difficilement évaluable d'une manière générale parce que "la qualité est toujours relative et s'énonce donc en termes de rapport entre un objet sur lequel on recueille des informations, le référé, et un référentiel qui sert de critère pour la comparaison » (De Ketele \& Gérard, 2007). Néanmoins, la qualité est associée à l'efficacité des actions entreprises afin d'assurer la réussite des étudiants et à la capacité à décrire cette efficacité.

Ainsi, d'une manière générale, l'évaluation des enseignements et des formations a pour but de mesurer leur efficacité, celle-ci se définissant comme la capacité à atteindre les objectifs prédéfinis qui donnent du sens à l'action de formation et d'apprentissage. Or, c'est "en considérant les effets d'une action que l'on en vient à en apprécier l'efficacité " (Fleisthammel \& Massot, 2005). Et la mesure de l'efficacité est le fondement de tous les dispositifs d'amélioration de la qualité par l'identification des écarts entre les objectifs annoncés et les réalisations. Cette notion d'efficacité a été précisée dans le domaine de l'enseignement scolaire par Lessard et Meirieu (2005) qui y associent les performances des élèves ainsi que l'équité pédagogique : « II ne s'agit pas seulement d'être efficace, mais aussi

11 Plante, J., (2002) Conférence donnée au 15ème colloque international de l'ADMEE-Europe, La qualité dans la formation et l'enseignement, comment la définir, comment l'évaluer ? Lausanne.

12 [http://www.cne-evaluation.fr/fr/present/interstandardsENQA.htm] 
Evaluer les enseignants et les formateurs

Comment, pourquoi, pour quoi ?

équitable. II s'agit non seulement que l'ensemble du groupe d'élèves progresse, mais aussi que le fossé qui, au départ, sépare les élèves les plus faibles des plus forts ne se creuse pas et, si possible, se réduise ".

Nous postulons qu'un apprenant a une vision sans doute plus égocentrée des objectifs et qu'il portera plus d'intérêt à la notion d'efficacité qu'à celle d'équité. Afin de la mesurer, l'évaluation devra selon Dejean (2007) porter sur un triple objet qui distingue :

- les effets (prévus ou imprévus) de l'enseignement,

- les actions mises en place pour les produire,

- la recherche d'un lien de causalité entre actions et effets.

Le premier renvoie aux acquis et à l'efficacité pédagogique, le deuxième interroge les logiques de co-production du service. Mais c'est le troisième qui véritablement donne du sens à la démarche d'évaluation des enseignements.

Cette réflexion sur les objets de l'évaluation vise à déterminer ce qu'il convient d'évaluer par rapport aux objectifs prédéfinis, rappelés par l'ENQA : permettre aux enseignants de bénéficier d'un retour d'appréciation sur la qualité de leurs activités d'enseignement afin de l'améliorer. II s'agit donc d'une évaluation formative visant à interroger et faire évoluer leurs pratiques didactiques.

Gilles et al. (2007) ont insisté sur les actions et liens de causalité en proposant un outil de diagnostic à travers le modèle dit du polygone des paramètres de l'action didactique, qu'ils fondent sur un modèle didactique permettant à la régulation didactique de se mettre en place. Ils listent ainsi dix objets d'évaluation qu'ils placent aux dix angles du polygone pour les relier entre eux afin d'illustrer l'entrelacs des relations causales.

Cette complexité ainsi représentée vise notamment à démontrer la relativité des appréciations portées et les limites d'une utilisation des résultats autre que formative.

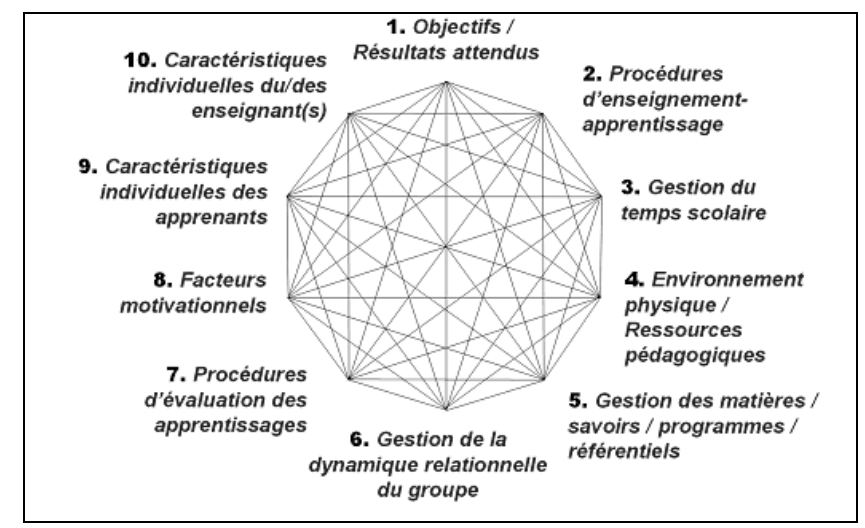

Fig. 1 : Polygone des paramètres des actions didactiques

Concernant les effets, ils ont été listés par Gérard (2003), dans le domaine de la formation continue, qui distingue les acquis, le transfert en milieu professionnel qui génère les compétences, et l'impact qui peut être vu comme l'effet produit au sein de l'organisation employeur. La satisfaction des apprenants n'apparaît pas explicitement dans ce cadre mais 
nous pouvons estimer qu'elle devrait découler en grande partie de l'efficacité dès lors que nous admettons que cela correspond à leurs propres objectifs.

Par ailleurs, si la satisfaction des acteurs et bénéficiaires des services de formation doit être évaluée régulièrement sous une forme à construire, la multiplicité des objectifs et des cibles des universités rend plus délicate la mesure de cette satisfaction, qui peut être immédiate ou différée. De même qu'une entreprise peut être écartelée entre les attentes de ses clients, de ses actionnaires et de ses salariés, l'Université doit arbitrer entre les intérêts des étudiants, de son personnel, du marché et des employeurs. A ce titre, nous devons considérer que la maximisation de la satisfaction immédiate des apprenants ne peut pas être le seul objectif de d'un établissement relevant du service public.

Cela pose bien entendu la question de l'évaluation des enseignements par les étudiants (EEE) et de la validité de ses effets sur les pratiques des susdits enseignants. Dans le cadre d'une étude réalisée dans une université du Québec, Thivierge et Bernard (1996) ont étudié les représentations des étudiants à propos de l'importance et de l'utilité de l'évaluation de l'enseignement et de sa prise en compte par l'institution. Les étudiants interrogés estiment à $95 \%$ qu'ils sont incontournables lorsqu'il s'agit d'évaluer la qualité des enseignements et considèrent à $96 \%$ que cela peut permettre d'améliorer la qualité de la formation; mais ils ne sont plus que $84 \%$ à penser que cela est susceptible d'aider un enseignant donné à améliorer la qualité de ses interventions personnelles.

Une analyse très complète de la littérature dans le domaine de l'impact sur les pratiques enseignantes a été développée par Détroz (2007) qui relève les contradictions fortes entre les auteurs et qui conclut qu' « en toute objectivité, on peut, tout au plus, parler d'un faisceau d'arguments qui plaident pour la validité de l'EEE ".13 Par précaution et en raison des biais constatés, il suggère alors de ne l'utiliser qu'à des fins formatives et non administratives. II se réfère dans ce cadre aux affirmations de Kulik (2001) qui souligne le caractère partiel et donc imparfait de la preuve de la validité de ce type de mesure.

Néanmoins, dans le cadre d'une enquête menée auprès de soixante-deux universités européennes, le quatrième rapport Trends de l'EUA souligne que "la conclusion la plus significative en matière de participation étudiante est le fait que les institutions qui connaissent et encouragent une participation dynamique de leurs étudiants ne rencontrent pas de problème de prise en compte des critiques, des plaintes et des recommandations concernant l'enseignement. L'inverse a été constaté pour les établissements dont les étudiants ne sont pas impliqués dans les processus de qualité (un quart de l'échantillon) „14. De ce point de vue, l'évaluation de l'enseignement par les étudiants peut devenir un outil de régulation.

Nous concluons de cette approche théorique que l'évaluation des enseignements par les étudiants est un élément incontournable du processus d'amélioration de la qualité mais qu'il convient d'être prudent quant à l'utilisation des résultats obtenus. Les appréciations obtenues restent au niveau des représentations. Ce qui n'enlève rien à leur intérêt, puisque les

\footnotetext{
${ }^{13}$ Detroz, P. (2007). Conférence sur l'Evaluation des enseignements : de la contrainte administrative à l'amélioration des pratiques, Fribourg, 24/09/2007.

${ }^{14}$ Reichert, S. \& Tauch, C., (2005), Etat de la mise en œuvre des réformes de Bologne par les Universités européennes, quatrième rapport trends de l'EUA, [http://www.eua.be/eua/jsp/en/upload/ TrendsIV_FINAL_FR.1128074006210.pdf].
} 


\section{Evaluer les enseignants et les formateurs}

Comment, pourquoi, pour quoi ?

étudiants, coproducteurs de ce service, raisonnent et agissent à partir de leurs représentations.

\section{Choix méthodologiques}

De préférence à d'autres outils, impliquant également les étudiants comme les entretiens de groupe, l'analyse de leurs performances, le portfolio, ... nous avons retenu la méthode du questionnaire, plus adapté à une mise en place au niveau de l'université dans son ensemble. Mais, comme on l'a vu dans ces lignes, ces questionnaires de satisfaction sont en général l'œuvre des enseignants responsables de diplômes ou de filières, quand ils ne sont pas rédigés par l'administration d'un département. Dans notre recherche, nous avons décidé d'opter pour une démarche plus novatrice, qui associe les étudiants dès la construction du dispositif d'évaluation.

Nous reprenons en cela l'idée qu'évaluer, c'est connaître pour construire ensemble du sens : si l'usager d'un service a une influence réelle, au travers de son comportement, sur le résultat du processus (ici, d'apprentissage), l'associer d'emblée au travail d'ingénierie du dispositif permettra, à l'issue de l'année de cours, de lui soumettre des outils d'appréciation qui ont une signification pour lui. Les critères de qualité des enseignements et des formations que les apprenants retiennent ne sont pas forcément ceux que les enseignants peuvent imaginer a priori, nous le verrons d'ailleurs dans plusieurs exemples ci-dessous.

Notre recherche a par conséquent opté pour la démarche suivante: dans chaque composante de notre université (UFR, IUT, Écoles d'Ingénieurs ${ }^{15}$ ), deux panels d'une dizaine d'étudiants ont été composés, l'un regroupant des néophytes, l'autre des apprenants plus chevronnés (L1 et M1 pour les UFR, DUT1 et Licence Professionnelle pour les IUT, $1^{\circ}$ et $3^{\circ}$ années de formation pour les Écoles). Les caractéristiques de cette population d'étude nous permettront par la suite des études comparatives: primo-arrivants versus anciens, filières sélectives versus UFR, formations générales versus formations professionnalisantes.

Les interviews ont été menées sous forme de focus group, l'idée étant de faire expliciter les attentes de groupes homogènes tout en autorisant des interactions directes entre leurs membres. On espère de la sorte parvenir à un approfondissement des positions, à une explicitation des présupposés de chacun lorsqu'il est confronté à un pair qui le contredit. Le résultat obtenu est ainsi plus riche que dans le cas d'interviews en face à face.

Conformément aux objectifs annoncés, les entretiens semi-directifs ont abordé les trois thèmes suivants, du point de vue des étudiants :

-quels sont les signes de qualité d'un enseignement (contenus, forme, structure générale)?

-quels sont les signes de qualité d'une formation (contenus, organisation, compétences construites)?

- de quelle manière devront être pris en compte les résultats des futurs questionnaires de satisfaction (destinataires)?

La logique naturelle de Grize $(1990,1996)$ a été retenue comme outil d'analyse du corpus ainsi réuni. Elle nous a semblé pertinente en ce qu'elle se fixe pour objectif de rendre compte de la construction des schématisations discursives, des raisonnements non formels

${ }^{15}$ Compte tenu des dates de rentrée, ce public n'a pas encore pu être interrogé au moment de la rédaction du présent article. 
dans l'interaction verbale orale. Une schématisation n'est pas le reflet exact et définitif d'une réalité préexistante, mais seulement un point d'entente vers lequel les énonciateurs coprésents convergent. En effet, les mots lorsque nous les utilisons sont déjà porteurs d'une signification sur laquelle nous nous entendons peu ou prou. A partir de cet accord a minima, des tractations s'engagent pour stabiliser le sens que les interlocuteurs désirent donner aux objets ainsi manipulés (nos trois thèmes d'entretien). Ce travail de réaménagement des contenus ne s'effectue bien souvent que sous la pression d'autrui, à travers les diverses figures de l'argumentation. Un faisceau d'objet est de la sorte progressivement négocié et construit. II regroupe les aspects que l'on considère finalement comme rattachés à cet objet : des propriétés, des relations et des schèmes d'action.

\section{5. Étude empirique}

Voyons pour commencer comment se construit la notion de "Qualité de l'Enseignement ", à quels signes les étudiants envisagent de se raccrocher pour en juger dans le questionnaire de satisfaction de fin d'année universitaire.

Ce qui apparaît en premier lieu, c'est que, de façon tout à fait constante d'un panel à l'autre, ils s'estiment peu légitimes en tant que critiques des contenus dispensés: "Pour l'instant, on n'a pas une vision globale » (L1), " II est difficile pour nous d'évaluer la qualité des contenus : tous sont nouveaux pour nous " (LPro), "Le prof ne doit pas meubler, il doit trier et organiser les informations disponibles " (M1).

Par contre, ils considèrent qu'ils sont compétents pour juger de la forme et du plan d'ensemble d'un cours, puisqu'ils ne sont conçus que pour eux, dans le seul but de leur faciliter les apprentissages. On ne peut que leur donner raison sur ce point et donc, par voie de conséquence, accepter à l'avance de tenir compte de leurs remarques dans ce domaine.

Les propriétés d'un bon cours sont alors sa structure logique, qui a pour corollaire la fluidité du propos de l'enseignant (L1) ; alors, "le temps passe vite, sans stress " (DUT1). L'information ainsi mise en scène se doit d'être d'actualité et non obsolète, principalement dans des domaines professionnels où elle évolue vite (LPro). Le scénario pédagogique retenu se doit de motiver les étudiants, en recourant à l'interactivité (LPro). Dans tous les cas, il convient de ne pas "prendre les étudiants de haut ", d'avoir un peu d'humour (DUT1), de les considérer comme des adultes (LPro).

La qualité d'un enseignement exige également certaines mises en relation. Nous avons déjà entrevu ci-dessus celle qui s'établit, toujours de l'avis des étudiants, entre la préparation magistrale préalable et l'assurance dont le professeur fait preuve au moment de sa prestation publique (L1; M1). Plusieurs groupes ont ensuite fait part, avec leurs mots à eux, des " périphériques » du cours : tantôt des documents complémentaires mis en ligne après la séance (LPro), tantôt des exemples concrets vus en Travaux Dirigés (DUT1), tantôt un plan de l'exposé et une bibliographie spécifique (L1). Pour autant, la séquence d'enseignement en présentiel semble indispensable aux interviewés : l'enseignant fait preuve d' " humanité ", il a de la considération pour ses étudiants, son "rayonnement" donne vie au cours, son «plaisir » est communicatif (L1; M1), toutes choses qu'une formation exclusivement à distance ne peut leur apporter de leur point de vue. Un groupe enfin évoque la relation entre enseignement et recherche... mais pour déplorer que certains enseignants préfèrent manifestement la recherche à la transmission de leurs savoirs (DUT1) : une telle question 


\section{Evaluer les enseignants et les formateurs \\ Comment, pourquoi, pour quoi?}

mérite assurément d'être retenue pour le document final, puisqu'il s'agit là ni plus ni moins que du statut de l'enseignant-chercheur.

La qualité de l'enseignement se traduit enfin à travers certains schèmes d'action. Un bon enseignant sait «enflammer la salle " (L1), il sollicite les étudiants, favorise les échanges (L1), mais tout en gardant le cap, sous peine de perdre la majorité de son groupe (M1). Réciproquement, l'étudiant fait la démarche d'aller vers l'enseignant, durant le cours et endehors (LPro) ${ }^{16}$, ce qui suppose que l'enseignant reste parfois disponible après les heures de cours, car « être prof, c'est aussi avoir un engagement relativement à sa discipline " (M1). De façon très significative, cette disponibilité des enseignants se traduit par l'importance de leur fournir des bureaux... dans l'esprit des étudiants des composantes dont c'est le cas (IUT), alors que cette idée n'est même pas évoquée par les autres Unités de Formation et de Recherche (UFR).

Passons maintenant à la "Qualité d'une Formation". II convient en préambule de souligner que les étudiants des différents groupes ont été interrogés pour savoir sur quels découpages temporels ils souhaitaient être questionnés. Si le Cours et la Formation (un an, deux ans, trois ans selon le cas; un semestre s'agissant des mobilités étudiantes dans le cadre du LMD) ont retenu leur attention, l'Unité d'Enseignement leur a semblé un échelon tout à fait factice, agrégat aléatoire construit dans un seul but d'évaluation sommative (DUT1).

Une "bonne » formation doit viser à une ouverture d'esprit (DUT1; M1) plus qu'à une adaptation stricte à une entreprise (DUT1). Elle doit respecter l'équilibre entre les différents champs d'une discipline (L1), et les descriptifs des cours doivent être aisément accessibles, surtout lorsqu'il s'agit d'options (L1).

Au niveau des relations, les étudiants ont surtout évoqué celles avec le monde professionnel, sous de multiples aspects. Une formation de qualité doit pour eux associer et en même temps distinguer Professeurs et Intervenants. Les premiers dispensent des contenus structurés, "à prendre en notes"; les seconds donnent "de bons conseils professionnels ", ils exposent des cas concrets, voire des anecdotes, "à mémoriser " (LPro). Les étudiants doivent avoir périodiquement le choix entre s'insérer dans le monde du travail ou poursuivre leur cursus (DUT1). Pour motiver cette décision, les stages en entreprise, voire des cursus en alternance (DUT1; LPro) sont des éléments de satisfaction. Ces périodes servent également à exercer des responsabilités sur un lieu de travail grâce aux apprentissages réalisés préalablement (LPro). Mais cette préoccupation est présente également chez les étudiants de L1 et de M1, dans une variante propre. Ils recensent trois façons de mettre leurs parcours à profit. Dans le premier cas, il s'agirait de valider un projet professionnel préexistant. Dans le second, un cursus bien conçu doit permettre d' "ouvrir des portes ", de suggérer de nouveaux horizons aux plus indécis, en leur présentant d'autres métiers (" pas seulement ceux de l'enseignement " ajoutent plusieurs d'entre eux). On va même jusqu'à proposer que les options de L1 correspondent systématiquement à l'ensemble des formations professionnalisantes du domaine (LPro, Master), afin de pouvoir se

\footnotetext{
${ }^{16}$ On remarquera au passage que c'est là l'une des très rares questions portant sur l'attitude des étudiants et non des enseignants : l'usager d'un service est partie prenante à sa réalisation, mais il n'a pas conscience de sa part de responsabilité. L'un des objectifs des questionnaires de satisfaction élaborés en commun est également de sensibiliser les étudiants dans ce domaine.
} 
Gangloff, Weisser, Bennaghmouch, Ben Abid-Zarrouk

Construction d'un dispositif d'évaluation des enseignements et des formations

déterminer en connaissance de cause le moment venu. Dans le troisième, il s'agit surtout d'autoriser des réorientations à certains moments, de penser des passerelles d'un parcours à un autre.

Ces positions débouchent sur quelques propositions d'actions. De façon très logique, a été évoquée alors la question des compétences professionnelles. Une formation de qualité doit permettre l'explicitation des compétences validées (LPro), afin de rendre les diplômés employables (DUT1). Là encore, les étudiants en formation "générale » (UFR) ne sont pas en reste. Ils soulignent la difficulté qu'ils ont de rédiger un CV en vue d'une recherche d'emploi, ne sachant pas expliciter leurs acquis (M1)17. Ce qui les amène à prendre conscience du fait qu'ils ne sont pas suffisamment valorisés. Ils avancent alors l'idée de faire reconnaître officiellement et symboliquement leurs savoirs et savoir-faire, par une cérémonie de remise de diplômes par exemple. Leur but est de démontrer par là que la Licence n'est pas seulement un passage obligé vers les concours, les formations professionnelles ou les Masters, mais un acquis positif qui mérite d'être reconnu à sa juste valeur (M1).

Les points essentiels d'accord / de divergence entre filières générales ouvertes et filières professionnelles sélectives peuvent se résumer par le tableau suivant :



Tab. 1 : Accords et divergences entre filières générales et filières professionnelles

Les signes de qualité d'un Enseignement et d'une Formation ont été de la sorte repérés par les différents groupes d'étudiants, à partir desquels le questionnaire de satisfaction pourra être rédigé. Restait à réfléchir sur l'exploitation souhaitée des résultats de ces questionnaires.

La représentativité des opinions qui auront été ainsi recueillies a tout d'abord fait l'objet de quelques interventions de la part des étudiants interviewés. II vaut mieux s'en tenir aux réponses les plus fréquentes, statistiquement (L1), un problème ponctuel dans un cours donné n'est sans doute qu'un détail au sein de toute une année de formation (DUT1). De plus, si l'opération est reconduite d'année en année, il conviendra de modifier régulièrement le questionnaire au vu des progrès constatés, ce qui engagerait l'université dans une démarche de recherche de qualité constante et suivie (M1).

17 II est à noter que l'obligation qui est désormais faite à toute formation, même non professionnalisante, de rédiger sa fiche RNCP (Répertoire National de la Certification Professionnelle) devrait conduire à des progrès dans ce domaine. 


\section{Evaluer les enseignants et les formateurs \\ Comment, pourquoi, pour quoi ?}

Le premier destinataire qui est venu à l'esprit des interviewés était bien entendu l'enseignant. Mais il s'agit d'évaluer les enseignements et les formations... et non les professeurs. Aussi, ont-ils imaginé un certain nombre de relais susceptibles de filtrer l'information et de la présenter dans des formes acceptables: le chef de département, le directeur de composante (DUT1 ; LPro). Certains ont au passage insisté sur le fait que, si les étudiants ont besoin d'être motivés par leurs professeurs, l'inverse est vrai également: les points de satisfaction devront être signalés eux aussi, le renforcement positif a des effets bénéfiques (M1).

Un autre destinataire a été avancé, de façon plus surprenante peut-être : les lycéens. L'argument invoqué consiste à dire qu'ils feraient ainsi un choix plus rationnel de leur établissement, dans une région où trois pays et quatre universités sont à moins de quarante minutes de trajet. La concurrence existe de toute façon, mais les décisions reposent pour l'heure sur des avis isolés (M1).

\section{Conclusion}

II se dégage de ces entretiens un certain nombre de principes qui peuvent régir la rédaction du futur questionnaire de satisfaction. C'est là une phase encore à venir de nos travaux, à travers des négociations avec les directeurs de composantes et les enseignantschercheurs.

Ce qui tombe sous le sens pour tous les panels d'étudiants interrogés, c'est qu'un questionnaire par Cours s'impose. L'accord se fait spontanément sur ce point. Mais les entretiens mettent de plus en évidence que certains thèmes ne peuvent être interrogés que sur le plan plus global de la Formation.

Bien évidemment, les filières sélectives et professionnalisantes (IUT) ne sont pas sensibles exactement aux mêmes problèmes que les formations générales (UFR). De plus, les étudiants néophytes (L1, DUT1) n'ont pas la même expérience que leurs aînés (LPro, M1). Mais par-delà ces différences contextuelles, des thèmes transversaux ont pu être mis en évidence.

Au niveau des Cours:

- évaluer la forme plutôt que le contenu ;

- du point de vue de la forme, être sensible

- à la préparation préalable, qui rejaillit sur l'attitude du professeur,

- à l'accompagnement de la séance en présentiel, par une documentation spécifique et par la disponibilité des enseignants.

Au niveau des Formations :

- respecter les équilibres de contenus, de durée, ... ;

- porter attention au monde du travail

- en invitant des intervenants durant les cours,

- en intégrant des périodes de stage,

- en identifiant et reconnaissant les acquis, tout au long d'un cursus.

Au niveau de la diffusion des résultats :

- dédramatiser l'enquête, ne retenir que les grandes tendances ;

- être positif à chaque fois que possible ;

- assurer la publicité de l'établissement envers les lycées. 
Gangloff, Weisser, Bennaghmouch, Ben Abid-Zarrouk

Construction d'un dispositif d'évaluation des enseignements et des formations

Quelles peuvent être les qualités métrologiques que l'on est en droit d'attendre d'un tel futur outil?

La validité nous semble en être la principale qualité. En effet, les étudiants étant à l'origine des items qui leur seront soumis, notre enquête devrait bien mesurer ce pour quoi elle a été conçue. A l'inverse des documents rédigés par des enseignants ou des administratifs, ce projet s'appuie sur un panel représentatif des usagers eux-mêmes. II évite ainsi le biais engendré par la mise en abyme des représentations, celles que les enseignants se font des représentations des étudiants.

La fidélité ne constitue pas quant à elle un critère décisif. II s'agit uniquement à travers ce dispositif d'obtenir un instantané de la façon dont un cours ou une formation ont été vécus. La stabilité temporelle des réponses n'est en rien recherchée. Bien au contraire, on pourrait imaginer de renvoyer à un groupe d'étudiants les résultats qu'ils ont produits dans leurs questionnaires par rapport à un enseignement donné. Ceci dans le but de les rediscuter en acceptant par conséquent les uns et les autres de modifier à la fois nos représentations à la suite des éclaircissements fournis, et la formation elle-même à partir des arguments reconnus recevables. On retrouve là les aspects purement formatifs d'une telle évaluation.

On aura, pour finir, un aperçu de la sensibilité de l'outil au moment des premiers traitements quantitatifs. Cela se traduira par une attention toute particulière portée aux items discriminant les formations sélectives / non sélectives, les étudiants en formation générale / professionnelle, les inscrits en Licence / en Master. Mais aussi, et de façon symétrique, aux réponses qui font l'unanimité, quels que soient les critères différenciateurs pris en considération. On pourra de la sorte distinguer le générique (commun à tous les étudiants) du spécifique (à une filière donnée).

Un questionnaire de satisfaction n'est bien entendu qu'un outil parmi d'autres, qui se complètent mutuellement. S'agissant de l'évaluation interne, on citera pour mémoire l'évaluation de la qualité des maquettes de formation par le CEVU (Conseil des études et de la vie universitaire) de chacun de nos établissements, ou les différents indicateurs quantitatifs qui renseignent aussi bien sur les flux d'étudiants (parcours, orientations, ...) que sur leur devenir après obtention d'un diplôme, etc. L'évaluation externe est assurée actuellement au plan national par l'AERES (Agence d'évaluation de la recherche et de l'enseignement supérieur), dont les critères devraient progressivement s'affiner et se stabiliser. Ces pratiques divergent encore d'un pays à l'autre.

La mise en place des standards européens (ESG) a démontré, entre autres leçons importantes, qu'un bon équilibre doit être maintenu entre processus internes et externes de garantie de la qualité, qui doivent se soutenir mutuellement et contribuer chacun au développement d'une culture de la qualité au sein des établissements d'enseignement supérieur et à la construction et la mise en œuvre de systèmes de garantie de la qualité nationaux cohérents et intégrés. L'amélioration de la qualité dans l'enseignement supérieur résulte dans une large mesure des interactions et des relations entre différents acteurs et partenaires ${ }^{18}$. La construction concertée du questionnaire de satisfaction destiné aux étudiants est un premier pas dans ce sens.

\footnotetext{
${ }^{18}$ Document de politique d'ENQA sur la garantie de la qualité dans l'espace européen de l'enseignement supérieur à l'occasion de la conférence des ministres responsables de l'enseignement supérieur à Louvain-la-Neuve les 28 et 29 avril 2009.
} 
Evaluer les enseignants et les formateurs

Comment, pourquoi, pour quoi ?

\section{Bibliographie}

Aballéa, F. \& Bercovitz A. (1990). Pour une approche non dogmatique des démarchesqualité, Études et expérimentations en formation continue, 6, 41-47.

Barbier, J.-M. (1985). L'évaluation en formation. Paris : PUF.

Baslé, M. (2000). Évaluation des politiques publiques et gouvernance à différents niveaux de gouvernement, Cahiers Economiques de Bretagne, 2, 17-24.

Caby, F. \& Jambart, C. (2002). La qualité dans les services. Paris : Economica.

De Ketele, J.-M. \& Gerard, F.-M. (2007). La qualité et le pilotage du système éducatif. In M. Behrens, M. (Éd.). La Qualité en éducation. Pour réfléchir à la formation de demain (pp. 1938). Québec : Presses de l'Université.

Dejean, J. (2007). Les démarches qualité dans l'enseignement supérieur, entre évaluation et contrôle. In A. Heldenbergh (Ed.), Les démarches qualité dans l'enseignement supérieur en Europe. Paris : L'Harmattan, 15-54.

Detroz, P. (2007). Conférence : Evaluation de la qualité des enseignements : de la contrainte administrative à l'amélioration des pratiques, Centre de Didactique Universitaire de Fribourg (Suisse), 24/09 [www.smart.ulg.ac.be/smartweb/documents/fribourg2007/fribourg240907.pdf]. Feisthammel, D. \& Massot, P. (2005). Fondamentaux du pilotage de la performance. Saint Denis la Plaine : AFNOR.

Gérard, F.-M. (2002). L'indispensable subjectivité de l'évaluation, Antipodes, $n^{\circ} 156$, avril 2002, 26-34.

Gérard, F.-M. (2003) L'évaluation de l'efficacité d'une formation, Gestion 2000, 20(3), 13-33.

Gilles, J.-L. et al. (2007). Apports d'un modèle de paramétrage des actions didactiques et d'une approche qualité dans le contexte du CAPAES. In : Actes du 24ème congrès de l'Association Internationale de Pédagogie Universitaire (AIPU) : Vers un changement de culture en enseignement supérieur, 605-609.

Grize, J.-B. (1990). Logique et langage. Paris : Ophrys.

Grize, J.-B. (1996). Logique naturelle et communications. Paris : PUF.

Hoppe, R. (1999). "Policy analysis, science and politics: from speaking truth to power" to "making sense together". Science and Public Policy, 26(3), 201-210, june.

Kulik, J.A. (2001). Student rating: Validity, utility, and controversy. In M. Theall, P.C. Abrami \& L.A. Mets (Ed.), The student ratings debate: Are they valid? how can we best use them? San Francisco: Jossey Bass.

Lessard, C. \& Meirieu, Ph. (Ed.) (2005). L'obligation de résultats en éducation. Évolution, perspectives et enjeux internationaux. Bruxelles : De Boeck

Murray, H.G. (1984). The impact of formative and summative evaluations of teaching in north American universities. Assessment and Evaluation in Higher Education, 9(2), 117-132.

Plante, J. (2002). Conférence. 15ème colloque international de I'ADMEE-Europe. La qualité dans la formation et l'enseignement, comment la définir, comment l'évaluer?

Rapport EVALUE (1998). Évaluation et auto-évaluation des universités en Europe. Paris: Dubois.

Thivierge, A. \& Bernard, H. (1996). Les croyances des étudiants à l'égard de l'évaluation de l'enseignement. Mesure et évaluation en éducation, 18(3), 59-84. 
Gangloff, Weisser, Bennaghmouch, Ben Abid-Zarrouk

Construction d'un dispositif d'évaluation des enseignements et des formations

\begin{abstract}
Webographie
Agence pour l'évaluation de la qualité dans l'enseignement supérieur: [www.aeqes.be/ documents/AEQES_18.12.06._Bruno\%20Curvale.ppt/]

Comité national d'évaluation: [http://www.cne-evaluation.fr/fr/present/interstandardsENQA. $\mathrm{htm}]$

European university association: [http://www.eua.be/eua/jsp/en/upload/TrendsIV_FINAL_FR. 1128074006210.pdf]

Ministère de l'enseignement supérieur et de la recherche, e-DGESIP: [http://edges.sup. adc.education.fr/edes/extranet/edes/extranet/contrats/editorial_contrats/autoeval/autoE.htm]
\end{abstract}

